\title{
Der Einfluß der Anordnung von Schraubenversetzungen auf die Gitterwärmeleitfähigkeit bei tiefen Temperaturen
}

\author{
Peter Gruner * \\ Institut für Theoretische und Angewandte Physik der Technischen Hochschule Stuttgart \\ und Max-Planck-Institut für Metallforschung Stuttgart \\ (Z. Naturforschg. 20 a, 1626-1639 [1965] ; eingegangen am 7. August 1965)
}

\begin{abstract}
Mit Hilfe der nichtlinearen Elastizitätstheorie wird der thermische Widerstand durch Schraubenversetzungen, die in bestimmter Weise angeordnet sind, berechnet. Es zeigt sich dabei, daß in Kupfer oberhalb der Temperatur des flüssigen Heliums die Art der Anordnung keine Rolle spielt und der Wärmewiderstand der Versetzungsgruppe in guter Näherung gleich ist dem bei statistisch verteilten Versetzungen. Unterhalb von etwa $4^{\circ} \mathrm{K}$ findet man, daß die Wärmeleitfähigkeit stärker als quadratisch mit zunehmender Temperatur ansteigt.
\end{abstract}

\section{Einleitung}

Die Streuung der Gitterwellen an den Fehlstellen des Kristalls führt zu einem Wärmewiderstand, der wegen der für jeden Gitterfehlertyp spezifischen Wellenlängenabhängigkeit des Streuquerschnitts eine charakteristische Funktion der Temperatur ist. Bei regelloser Verteilung und Abständen der Gitterfehler, die groß gegenüber der effektiven Wellenlänge der Gitterschwingungen sind, setzt sich der Gesamtwiderstand additiv aus den Einzelwiderständen zusammen ${ }^{1}$. Diese Voraussetzung ist aber bei sehr tiefen Temperaturen nicht erfült, da die dominierenden Wellenlängen dann vergleichbar oder sogar größer als die Abstände der Fehlstellen sind, so daß der kohärente Anteil der Streuung nicht mehr vernachlässigt werden darf.

Es soll im folgenden untersucht werden, wie groß der Einfluß des kohärenten Beitrags der Streuung von Gitterwellen an Schraubenversetzungen verschiedener Anordnung auf die Zunahme des Wärmewiderstands ist, und von welcher Temperatur ab die Voraussetzung regelloser Verteilung der Fehlstellen nicht mehr gerechtfertigt werden kann. Zur Berechnung der Wechselwirkung der Gitterwellen mit dem Verzerrungsfeld der Versetzungen benützen wir die nichtlineare Elastizitätstheorie, was bei tiefen Temperaturen sicher erlaubt ist. Die Wahrscheinlichkeit für die Vernichtung eines Phonons der Welle mit

* Dissertation, Technische Hochschule Stuttgart 1965.

1 Im vorliegenden Fall vernachlässigen wir die Dreiphononenprozesse, da wir uns auf so tiefe Temperaturen beschränken, bei denen die Gitterwellen im wesentlichen nur durch Versetzungen gestreut werden (siehe H. Bross, P. Gruner u. P. Kirschenmann, Z. Naturforschg. 20 a, 1611 [1965], voranstehend).
Ausbreitungsvektor $\mathfrak{f}$ und Polarisation $j$ ( $e_{\mathfrak{k}}^{j}$ : Einheitsvektor der Polarisation, $j=1,2,3$ ) und die gleichzeitige Erzeugung eines Energiequants der Welle $\left(\mathfrak{f}^{\prime}, j^{\prime}\right)$ ist dann vollständig durch das von den Gitterfehlern erzeugte Gesamtverzerrungsfeld bestimmt.

Das Transportproblem wird nach der in $\mathrm{I}^{2}$ angegebenen Methode mit Hilfe eines Variationsverfahrens gelöst. Aus Platzgründen können wir hier nicht den gesamten Formalismus zur Berechnung der Wärmeleitfähigkeit darstellen. Wir beziehen uns deshalb des öfteren auf die in I ausführlich dargestellte allgemeine Methode und verwenden die dort angegebenen Gleichungen.

Vergleicht man die in II $^{3}$ mitgeteilten theoretischen Ergebnisse des Wärmewiderstands einer Einzelschraubenversetzung mit den experimentellen Daten, die von Kemp, Klemens und Tainsh ${ }^{4}$ für Kupferlegierungen angegeben wurden, so erhält man unter der Voraussetzung regellos verteilter Versetzungen verhältnismäßig gute Übereinstimmung. Der theoretische Wärmewiderstand pro Versetzungslinieneinheit ist etwa um einen Faktor 2 kleiner als der experimentelle. Sehr viel schlechter ist die Übereinstimmung in Ionenkristallen. Der experimentelle Wert des Wärmewiderstands je Versetzungslinieneinheit ist hier etwa tausendmal größer als der theoretische (die Versetzungsdichte wurde bei den Ex-

2 H. Bross, Phys. Status Solidi 2, 481 [1962].

${ }^{3}$ H. Bross, A. Seeger u. P. Gruner, Ann. Phys. Leipzig (7), 11, 230 [1963].

4 W. R. G. Kemp, P. G. Klemens u. R. J. Tainsh, Phil. Mag. 4, 845 [1959]. 
perimenten mit Hilfe der Ätzgrübchenmethode bestimmt ${ }^{5,6}$ ). Aus den folgenden Untersuchungen ergibt sich, daß diese Diskrepanz zwischen Beobachtung und Rechnung nicht wesentlich vermindert werden kann, wenn man die Art der Versetzungsanordnung berücksichtigt.

\section{Die Streuung von Gitterwellen an einer Reihe von $N$ zur $z$-Richtung paralleler Schrauben- versetzungen}

Die Verteilungsfunktion $N_{0}^{j}$ der Phononenzustände im thermischen Gleichgewicht wird durch ein äußeres Temperaturgefälle so lange verändert, bis sich durch die Streuung der Gitterwellen an den Fehlstellen eine neue stationäre Verteilung $N_{\mathrm{t}}^{j}$ einstellt, die durch die linearisierte Boltzmannsche Transport- gleichung bestimmt ist. Während die Energieabhängigkeit von $\bar{N}_{\sharp}^{j}$ bei der Streuung von Gitterwellen an statischen Fehlstellen exakt bestimmt werden kann, läßt sich im allgemeinen diese Integralgleichung in bezug auf die Winkelabhängigkeit von $\bar{N}_{\mathrm{l}}^{j}$ nur näherungsweise lösen, da die Streuung - sieht man von Punktfehlern ab - nicht isotrop erfolgt. Man führt deshalb die Boltzmann-Gleichung nach I auf ein Extremalproblem zurück und löst dieses mit dem Ritzschen Verfahren, indem man die Entwicklungskoeffizienten eines geeignet gewählten Ansatzes für die Abweichung der Verteilungsfunktion im stationären Zustand von der im thermischen Gleichgewicht variiert. Man erhält dann ein lineares Gleichungssystem der Form

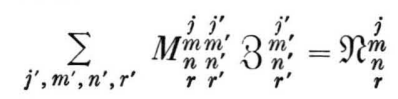

für die durch ${ }^{7}$

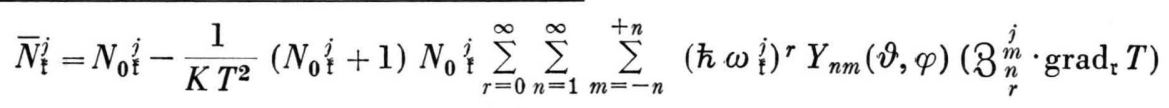

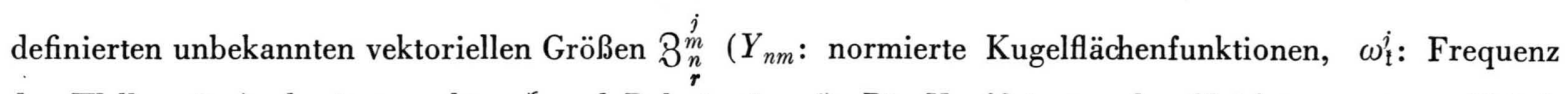
der Welle mit Ausbreitungsvektor $\mathfrak{f}$ und Polarisation j). Die Koeffizienten des Gleichungssystems (2.1a) sind dabei definiert durch den die gesamte Information über den Streuvorgang enthaltenden Ausdruck ${ }^{8}$

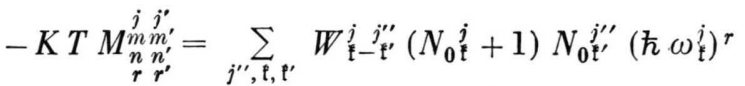

$$
\begin{aligned}
& \cdot Y_{n m}(\vartheta, \varphi)\left\{\delta^{j j^{\prime}}\left(\hbar \omega_{\mathrm{f}}^{j}\right) r^{r^{\prime}} Y_{n^{\prime} m^{\prime}}(\vartheta, \varphi)-\delta^{j^{\prime} j^{\prime \prime}}\left(\hbar \omega \hat{\psi}^{i^{\prime}}\right) r^{r^{\prime}} Y_{n^{\prime} m^{\prime}}\left(\vartheta^{\prime}, \varphi^{\prime}\right)\right\}
\end{aligned}
$$

mit der Symmetrieeigenschaft

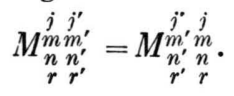

Die Wahrscheinlichkeit dafür, daß pro Zeiteinheit ein Phonon der Welle $(\mathfrak{f}, j)$ vernichtet und gleichzeitig eines der Welle $\left(\mathfrak{f}^{\prime}, j^{\prime}\right)$ erzeugt wird, hängt vom Quadrat der Fourier-Transformierten des Verzerrungstensors der Gitterfehler ab und ist bei der Streuung von Gitterwellen an statischen Fehlstellen nur dann von Null verschieden, wenn die Energie des emittierten Phonons gleich der des absorbierten ist. Für den Tensor der Gitterwärmeleitfähigkeit findet $\operatorname{man}^{2}$

$$
\boldsymbol{x}=-\frac{1}{T V_{0}} \sum_{i, m, n, r} \underset{r}{\underset{r}{m} \underset{r}{j} \Re_{r}^{j}} \underset{r}{j}
$$

5 R. Sproull, M. Moss u. H. W. Weinstock, J. Appl. Phys. 30, 334 [1959].

6 S. Ishioka u. H. Suzuki, J. Phys. Soc. Japan 18, Suppl. II, 93 [1963].

7 Der Wellenvektor $\mathfrak{k}$ ist durch die Frequenz $\omega_{\mathfrak{k}}^{j}$ und die sphärischen Winkel $(\vartheta, \varphi)$ festgelegt. mit

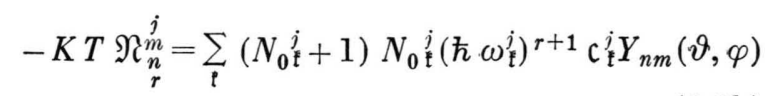

$$
\text { ( } V_{0}: \text { Kristallvolumen). }
$$

\section{a) Die Übergangswahrscheinlichkeit}

Das Gesamtverzerrungsfeld $\boldsymbol{\epsilon}_{\mathrm{R}}(\mathrm{r})$ einer Reihe von Versetzungen entlang der $x$-Achse kann in der Näherung der Elastizitätstheorie dritter Ordnung ${ }^{9}$ durch lineare Superposition der Verzerrungen der Einzelversetzungen $\boldsymbol{\epsilon}_{\mathrm{S}}(\mathfrak{r})$ dargestellt werden:

$$
\boldsymbol{\epsilon}_{\mathrm{R}}(\mathfrak{r})=\sum_{i=1}^{N} \boldsymbol{\epsilon}_{\mathrm{S}}\left(\mathfrak{r}-\dot{\mathfrak{i}}_{1} x_{i}\right)
$$

8 (2.1 b) geht aus I, $(5.21 \mathrm{c})$ hervor, wenn man dort den Beitrag der Normalprozesse wegläßt.

9 Wir gehen in der Entwicklung der potentiellen Energie nach den Verzerrungen bis zu Gliedern, die kubisch in den Verzerrungen sind. 
$\left(x_{i}:\right.$ Ortskoordinate der $i$-ten Versetzung, $i_{1}:$ Koordinateneinheitsvektor). $\boldsymbol{\epsilon}_{\mathbb{S}}(\mathfrak{r})$ ist der Verzerrungstensor der Elastizitätstheorie 2. Ordnung einer sich im Ursprung des Koordinatensystems befindenden Versetzung. Ist

$\boldsymbol{\epsilon}_{\mathrm{S}}(\mathfrak{g})=\frac{1}{(2 \pi)^{3 / 2}} \int \boldsymbol{\epsilon}_{\mathrm{S}}(\mathfrak{r}) \exp \{-\mathfrak{i} \mathfrak{g} \cdot \mathfrak{r}\} \mathrm{d} \tau_{\mathfrak{r}}$

die Fourier-Transformierte des Verzerrungstensors der Einzelversetzung, so erhält man aus (2.4a) die Fourier-Transformierte des Verzerrungstensors der gesamten Reihe:

$$
\boldsymbol{\epsilon}_{\mathrm{R}}(\mathfrak{g})=\boldsymbol{\epsilon}_{\mathrm{S}}(\mathfrak{g}) \sum_{i=1}^{N} \exp \left\{-\mathfrak{i} g_{1} x_{i}\right\} .
$$

In einem isotropen elastischen Kontinuum sind die einzigen von Null verschiedenen Komponenten von $\boldsymbol{\epsilon}_{\mathrm{S}}(\mathrm{g})$ bei einer zur $z$-Richtung parallelen Schraubenversetzung nach II:

$\varepsilon_{\mathrm{S} 13}(\mathfrak{g})=-\frac{1}{2} \frac{\mathfrak{i} b}{(2 \pi)^{1 / 2}} \frac{g_{2}}{g_{1}{ }^{2}+g_{2}{ }^{2}} \delta\left(g_{3}\right)=\varepsilon_{\mathrm{S} 31}(\mathfrak{g})$,

$\varepsilon_{\mathrm{S} 23}(\mathfrak{g})=\frac{1}{2} \frac{\mathfrak{i} b}{(2 \pi)^{1 / 2}} \frac{g_{1}}{g_{1}{ }^{2}+g_{2}{ }^{2}} \delta\left(g_{3}\right)=\varepsilon_{\mathrm{S} 32}(\mathfrak{g})$

( $b$ : Betrag des Burgers-Vektors).

Setzen wir (2.4c) in den in I angegebenen Ausdruck für die Übergangswahrscheinlichkeit ein und berücksichtigen, daß die Dilatation einer Schraubenversetzung in der Elastizitätstheorie 2. Ordnung nach (2.5a und b) Null ist, so finden wir

$$
W_{\mathrm{R} \mathfrak{t}-\mathfrak{t}^{\prime}}^{j j^{\prime}}=W_{\mathrm{S} \mathfrak{k}-\mathfrak{k}^{\prime}}^{j} F\left(\left|k_{x}-k_{x}{ }^{\prime}\right|\right)
$$

mit dem Anordnungsfaktor

$$
\begin{aligned}
& F\left(\left|k_{x}-k_{x}{ }^{\prime}\right|\right)=\sum_{i=1}^{N} \sum_{i^{\prime}=1}^{N} \exp \left\{\dot{\mathrm{i}}\left(k_{x}-k_{x}{ }^{\prime}\right) R_{i i^{\prime}}\right\}, \\
& \text { wobei } \quad R_{i i^{\prime}}=x_{i}-x_{i^{\prime}} \quad \text { ist }
\end{aligned}
$$

und

$$
\begin{aligned}
& W_{\mathrm{S} \mathfrak{t}-\mathfrak{f}^{\prime}}^{j}=\frac{(2 \pi)^{4}}{16\left[V_{0} \varrho_{0}\right]^{2}} \frac{\delta\left(\omega_{\mathfrak{t}}^{j}-\omega_{\mathfrak{k}^{\prime}}^{j^{\prime}}\right)}{\left(\omega_{\mathfrak{t}}^{i}\right)^{2}} \cdot \mid\left(e_{\mathfrak{t}}^{j} \cdot e_{\mathfrak{t}^{\prime}}^{j^{\prime}}\right) \mathfrak{f} \cdot \boldsymbol{\epsilon}_{\mathrm{S}}(\mathfrak{g}) \cdot \mathfrak{f}^{\prime}\left(4 A_{2}-A_{5}\right) \\
& +\left[\left(e_{\mathfrak{f}}^{j} \cdot \mathfrak{f}\right) e_{\mathfrak{t}^{\prime}}^{j^{\prime}} \cdot \boldsymbol{\epsilon}_{\mathrm{S}}(\mathfrak{g}) \cdot \mathfrak{f}^{\prime}+\left(e^{j_{\mathfrak{k}}^{\prime}} \cdot \mathfrak{f}^{\prime}\right) e_{\mathfrak{f}}^{j} \cdot \boldsymbol{\epsilon}_{\mathrm{S}}(\mathfrak{g}) \cdot \mathfrak{f}\right]\left(-8 A_{1}-4 A_{2}+2 A_{4}+2 A_{5}\right) \\
& +\left.\left[\left(e_{\mathfrak{f}}^{j} \cdot \mathfrak{l}^{\prime}\right) \mathfrak{f} \cdot \boldsymbol{\epsilon}_{\mathrm{S}}(\mathfrak{g}) \cdot e_{\mathfrak{t}^{\prime}}^{j^{\prime}}+\left(\mathfrak{e}_{\mathfrak{t}^{\prime}}^{j^{\prime}} \cdot \mathfrak{f}\right) \mathfrak{f}^{\prime} \cdot \boldsymbol{\epsilon}_{\mathrm{S}}(\mathfrak{g}) \cdot e_{\mathfrak{f}}^{j}+\left(\mathfrak{f} \cdot \mathfrak{f}^{\prime}\right) e_{\mathfrak{f}^{\prime}}^{j^{\prime}} \cdot \boldsymbol{\epsilon}_{\mathrm{S}}(\mathfrak{g}) \cdot e_{\mathfrak{k}}^{j}\right]\left(2 A_{2}-A_{5}\right)\right|^{2}
\end{aligned}
$$

mit $\quad \mathfrak{g}=-\left(\mathfrak{f}-\mathfrak{f}^{\prime}\right) \quad\left(\varrho_{0}\right.$ : Massendichte $)$.

Zur weiteren Diskussion des Anordnungsfaktors $F$ formen wir (2.6b) noch etwas um. Es ist

$$
\boldsymbol{F}=N+\sum_{i=1}^{N} \sum_{\substack{i^{\prime}=1 \\ i \neq i^{\prime}}}^{N} \exp \left\{\mathfrak{i}\left(k_{x}-k_{x}{ }^{\prime}\right) R_{i i^{\prime}}\right\} \text {. }
$$

Wenn die Versetzungen auf der $x$-Achse regellos verteilt und ihre Abstände groß sind gegenüber der dominierenden Wellenlänge der Gitterschwingungen, so ist der zweite Summand in (2.6f) bei großer Anzahl $N$ der Versetzungen eine rasch oszillierende Funktion und kann vernachlässigt werden. In diesem Fall dürfen die Übergangswahrscheinlichkeiten der Einzelstreuprozesse einfach addiert werden, was dann zum Gesamtwiderstand $N \varrho_{\mathrm{S}}$ führt $\left(\varrho_{\mathrm{S}}:\right.$ der durch eine Einzelversetzung erzeugte thermische Widerstand). Dagegen ist bei sehr tiefen Temperaturen $(T \approx 0)$ und nahe benachbarten Versetzungen $\left(k R_{i i^{\prime}} \approx 0\right.$ ) der Anordnungsfaktor $F \approx N^{2}$. Der thermische Widerstand der Reihe wird deshalb zwischen den Extremwerten $N \varrho_{\mathrm{S}}$ und $N^{2} \varrho_{\mathrm{S}}$ liegen.

Wie in II definieren wir die zueinander orthogo. nalen Polarisationseinheitsvektoren $e_{\ddagger}^{j}$ so, daß der transversale Polarisationsvektor $e_{\mathfrak{t}}^{\mathrm{T}_{2}}$ in einer Ebene senkrecht zur $z$-Richtung liegt. $\mathrm{e}_{\ddagger}^{\mathrm{L}}$ soll parallel zum Ausbreitungsvektor sein. Diese Forderungen werden durch

$$
\begin{aligned}
\mathfrak{e}_{\mathfrak{f}}^{\mathrm{L}} & =\mathfrak{f} /|\mathfrak{f}| ; \\
\mathfrak{e}_{\mathfrak{f}}^{\mathrm{T}_{1}} & =\frac{1}{|\mathfrak{f}|\left(k_{1}{ }^{2}+k_{2}{ }^{2}\right)^{1 / 2}}\left(\begin{array}{cc}
-k_{3} & k_{1} \\
-k_{3} & k_{2} \\
k_{1}^{2}+k_{2}^{2}
\end{array}\right) ; \\
\mathfrak{e}_{\mathfrak{f}}^{\mathrm{T}_{2}} & =\frac{1}{\left(k_{1}{ }^{2}+k_{2}{ }^{2}\right)^{1 / 2}}\left(\begin{array}{r}
k_{2} \\
-k_{1} \\
0
\end{array}\right)
\end{aligned}
$$

erfüllt. Wegen dieser Festsetzung und der Invarianz des Verzerrungstensors einer Schraubenversetzung mit dem Burgers-Vektor in Richtung $z$ gegen eine beliebige Drehung um die $z$-Achse, hängt $W_{\mathrm{St}^{j}-\mathfrak{t}^{\prime}}^{j^{\prime}}$ nur vom Differenzwinkel $\left(\varphi^{\prime}-\varphi\right)$ ab, was zur Folge hat, daß im Fall der Einzelschraubenversetzung nur die Matrizen $\begin{gathered}j \\ \substack{j \\ j^{\prime} \\ n m^{\prime} \\ r n^{\prime} \\ r r^{\prime}}\end{gathered}$ von Null verschieden sind, für die $m+m^{\prime}=0$ ist. Diese Symmetrieeigenschaft der

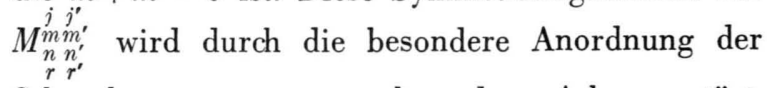
Schraubenversetzungen entlang der $x$-Achse zerstört. Da jedoch der Anordnungsfaktor $F$ nur vom Betrag 
der Differenz der $x$-Komponenten von $\mathfrak{H}^{\prime}$ bzw. $\mathfrak{f}^{\prime}$ abhängt und $W_{\mathrm{St}}^{j} j_{\mathbf{t}^{\prime}}^{j^{\prime}}$ die oben erwähnte Invarianzeigenschaft hat, ist $W_{\mathrm{R} \mathfrak{\xi}-\mathfrak{t}^{\prime}}^{j}$ invariant gegenüber Spiegelung an den Ebenen $k_{x}=0$ bzw. $k_{y}=0$. Infolgedessen

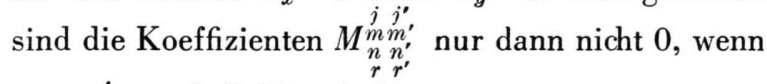
$m+m^{\prime}$ gerade Zahlen sind:

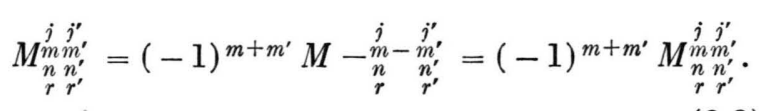

Im folgenden beschränken wir uns auf die Näherung $n=1$ in der Winkelabhängigkeit der Verteilungsfunktionen. $m$ kann dann nur die Werte $0,+1$

und -1 annehmen. Mit der Symmetrieeigenschaft (2.8) läßt sich das Gleichungssystem (2.1 a) in dieser Näherung in drei voneinander unabhängige Gleichungssysteme zerlegen:

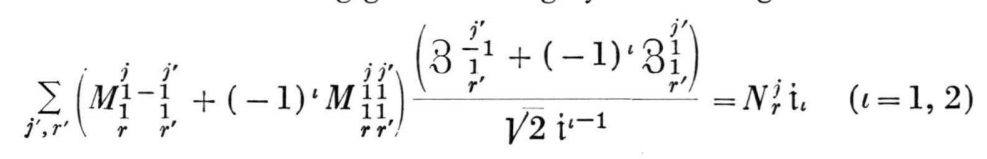

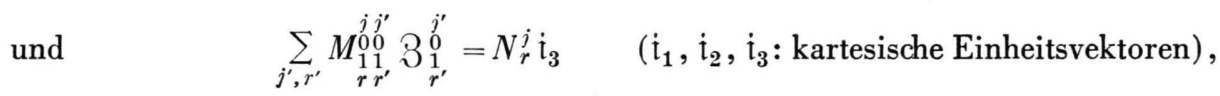

wobei

$$
N_{r}^{j}=-\left(\frac{4 \pi}{3}\right)^{1 / 2} \frac{V_{0}}{(2 \pi)^{3}} \frac{(K T)^{r+3}}{\hbar^{3}\left(c^{j}\right)^{2}}(r+3) ! \zeta(r+3) \quad \text { ist. }
$$

Die einzigen von Null verschiedenen Komponenten des Wärmeleitungstensors sind die Diagonalelemente

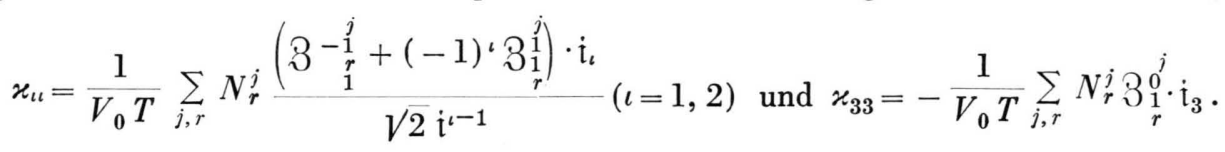

$$
\begin{aligned}
& \text { b) Berechnung der Matrixelemente } \begin{array}{c}
j \\
M_{1}^{j} m^{\prime} \\
1 \\
r \\
m^{\prime}
\end{array}
\end{aligned}
$$

Es wurde schon erwähnt, daß bei der Streuung der Gitterwellen an statischen Fehlstellen die Energie des emittierten Phonons gleich der des absorbierten sein muß. Außerdem bleibt nach (2.5a und b) die $z$-Komponente des f-Vektors bei der Streuung an Schraubenversetzungen mit Burgers-Vektor parallel zur $z$-Richtung erhalten. Nach der Integration über $|\mathfrak{f}|$ und $k_{z}{ }^{\prime}$ erhält man ${ }^{10}$

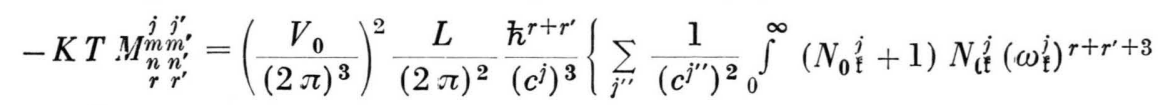

$\cdot \int_{0}^{\pi} \int_{0}^{2 \pi} \overline{W_{\mathfrak{S}}^{j}-\mathfrak{f}^{\prime}}\left[\delta^{j j^{\prime}} \Pi_{n^{\prime}}^{m^{\prime}}(\cos \vartheta)-\delta^{j^{\prime} j^{\prime \prime}} \Pi_{n^{\prime}}^{m^{\prime}}\left(\beta^{j^{\prime \prime} j} \cos \vartheta\right) \exp \left\{\mathfrak{i} m^{\prime} \psi\right\}\right] \Pi_{n}^{m}(\cos \vartheta)$

$\left.\cdot\left(\sum_{i=1}^{N} \sum_{i^{\prime}=1}^{N} \int_{0}^{2 \pi} \exp \left\{\dot{i} \frac{\omega_{\mathfrak{k}}^{j} R_{i i^{\prime}}}{c^{j}}\left[\sin \vartheta \cos \varphi-\left(\left(\beta^{i j^{\prime \prime}}\right)^{2}-\cos ^{2} \vartheta\right)^{1 / 2} \cos (\varphi+\psi)\right]\right\} \exp \left\{\dot{\mathfrak{i}}\left(m+m^{\prime}\right) \varphi\right\} \mathrm{d} \varphi\right) \sin \vartheta \mathrm{d} \vartheta \mathrm{d} \psi \mathrm{d} \omega_{\mathfrak{f}}^{j}\right\}$

In (2.11a) wurde benützt, daß

$$
\int_{0}^{\pi} F\left(\cos \vartheta^{\prime}\right) \delta^{2}\left\{\frac{\omega_{\mathfrak{k}}^{j}}{c^{j}} \cos \vartheta-\frac{\omega_{\mathfrak{k}}^{j}}{c^{j^{\prime \prime}}} \cos \vartheta^{\prime}\right\} \sin \vartheta^{\prime} \mathrm{d} \vartheta^{\prime}=\frac{L}{2 \pi} \frac{c^{j^{\prime \prime}}}{\omega_{\mathfrak{k}}^{j}} F\left(\beta^{j^{\prime \prime} j} \cos \vartheta\right)
$$

ist. Außerdem haben wir die kontinuumstheoretische Beziehung $\omega_{\mathfrak{k}}^{j}=c^{j}|\mathfrak{f}|$ verwendet und den Differenzwinkel $\psi=\varphi^{\prime}-\varphi$ eingeführt. Das Verhältnis der Schallgeschwindigkeit $c^{j} / c^{j^{\prime}}$ wurde durch $\beta^{j j^{\prime}}$ abge-

$10 \bar{W}_{\mathrm{S}}^{j}{ }_{\mathfrak{t}-\mathfrak{f}^{\prime}}^{j^{\prime}}$, erhält man aus $W_{\mathrm{S}_{\mathfrak{f}}-\mathfrak{k}^{\prime}}^{j}, i^{\prime}$, indem man die $\delta$-Funktionen über $\omega_{\mathfrak{f}}^{j}$ und $k_{z}$ durch 1 ersetzt, für $\left|\mathfrak{k}^{\prime}\right|$ und $\cos \vartheta^{\prime}$ die Werte $\omega_{\mathfrak{t}}^{\iota} / c j^{\prime}$ bzw. $\beta j^{\prime} j \cos \vartheta$ einführt. kürzt. $\Pi_{n}^{m}(\cos \vartheta)$ sind normierte zugeordnete Kugelfunktionen. Wegen den Symmetriebeziehungen (2.1c) und (2.8) brauchen wir nur diejenigen Matrixelemente zu berechnen, für die $c^{j} \geqq c^{j^{\prime}}$ ist.

An Hand von Gl. (2.11a) läßt sich für den Fall $n=1, m=m^{\prime}=0$ zeigen, daß

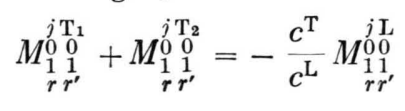


ist. Das hat zur Folge, daß die Determinante des Gleichungssystems $(2.9 \mathrm{~b})$ identisch gleich Null ist und daß deshalb die $z z$-Komponente des Wärmewiderstands wie bei einer Einzelschraubenversetzung verschwindet. Dieses Ergebnis war natürlich zu erwarten, da auch bei der Streuung der Gitterwellen an einer Gruppe von zur $z$-Richtung paralleler Schraubenversetzungen $k_{z}$ ungeändert bleibt.

Es sei schon an dieser Stelle darauf hingewiesen, daß wegen der Frequenzabhängigkeit des Anordnungsfaktors (2.6b) das Temperaturverhalten der Wärmeleitfähigkeit, zumindest bei tiefen Temperaturen, gegenüber demjenigen bei regelloser Versetzungsanordnung verändert wird.

Mit Hilfe der Methode der stationären Phase kann gezeigt werden ${ }^{11}$, daß für große Versetzungsabstände die Beiträge zu den Matrizen $M$ bei Übergängen zwischen longitudinalen und transversalen Phononenzuständen $\left(\mathrm{L} \rightleftarrows \mathrm{T}_{1}, \mathrm{~L} \rightleftarrows \mathrm{T}_{2}\right)$ wie $\left(R_{i i^{\prime}}\right)^{-2,5}$ gegen Null gehen, während bei Übergängen zwischen longitudinalen $(\mathrm{L} \rightarrow \mathrm{L})$ oder transversalen Zustän$\operatorname{den}\left(\mathrm{T}_{1} \rightarrow \mathrm{T}_{1}, \mathrm{~T}_{2} \rightarrow \mathrm{T}_{2}, \mathrm{~T}_{1} \gtrless \mathrm{T}_{2}\right)$ die Matrizen $M$ sich asymptotisch wie $\left(R_{i i}\right)^{-1}$ verhalten ${ }^{12}$. Eine $\mathrm{Ab}$ schätzung für sehr kleine Versetzungsabstände ergibt, daß bei $\mathrm{L} \rightleftarrows \mathrm{T}$-Übergängen erst unterhalb einer sehr tiefen Temperatur $T_{0}$ die Anordnung der Versetzungen eine Rolle spielt. Die Abschätzung ergibt für einen Versetzungsabstand von 20 Burgers-Vektoren in Kupfer den Wert $T_{0}=1,5^{\circ} \mathrm{K}$. Außerdem zeigte sich bei der Berechnung des Wärmewiderstandes einer Einzelschraubenversetzung (II), daß die Wahrscheinlichkeit für Übergänge der Art $\mathrm{L} \gtrless \mathrm{T}$ klein ist gegenüber derjenigen für Übergänge zwischen transversalen Phononenzuständen. Die eben angeführten Gründe berechtigen zur Vernachlässigung des zweiten Summanden in $(2.6 \mathrm{f})$ bei $\mathrm{L} \rightleftarrows \mathrm{T}$ Übergängen. Wir werden deshalb nur in den Fällen $\mathrm{L} \rightarrow \mathrm{L}$ und $\mathrm{T} \rightarrow \mathrm{T}\left(\beta^{i j^{\prime}}=1\right)$ den Phasenbeitrag berücksichtigen und bei $\mathrm{L} \rightarrow \mathrm{T}$-Übergängen die Versetzungen als regellos verteilt ansehen.

In (2.11a) ist nur der Integrand des letzten Faktors von $\varphi$ abhängig.

Für $\beta^{i j^{\prime \prime}}=1$ ist:

$$
I=\sum_{i=1}^{N} \sum_{i^{\prime}=1}^{N} \int_{0}^{2 \pi} \exp \left\{\mathrm{i} \frac{2 \omega \frac{\omega_{i}^{j}}{c^{j}} R_{i i^{\prime}}}{c^{j}} \sin \vartheta \sin \frac{\psi}{2} \sin \left(\varphi+\frac{\psi}{2}\right)\right\} \exp \left\{\mathrm{i}\left(m+m^{\prime}\right) \varphi\right\} \mathrm{d} \varphi .
$$

Das Integral von (2.13a) läßt sich mit der Substitution $\chi=\psi / 2+\varphi$ auf die Integraldarstellung der BesseLFunktion $J_{m+m^{\prime}}$ zurückführen. Man erhält:

$$
I=2 \pi(-1)^{m+m^{\prime}} \exp \left\{-\dot{i}\left(m+m^{\prime}\right) \psi / 2\right\} \sum_{i=1} \sum_{i^{\prime}=1} J_{m+m^{\prime}}\left(\frac{2 \omega_{i}^{j}}{c^{j}} R_{i i^{\prime}} \sin \vartheta \sin \psi / 2\right) .
$$

Die weitere Auswertung der Integrale (2.1la) ist an die spezielle Form der Koeffizienten $\bar{W}_{\mathrm{S}}^{j} \hat{k}_{-\mathfrak{k}^{\prime}}^{j^{\prime}}$ gebunden. Es würde zu weit führen, wollten wir die umf angreiche Berechnung jedes einzelnen Matrixelements hier darstellen. Wir wollen deshalb den weiteren Gang der Rechnung nur skizzieren. Die Untersuchungen

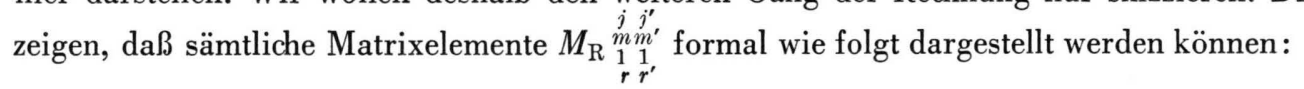

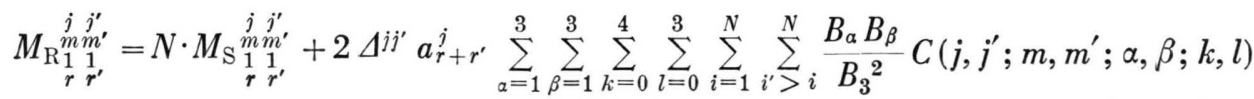

$$
\begin{aligned}
& \Im\left(m+m^{\prime}, r+r^{\prime}, k, l ; b_{i i^{\prime}}^{j}\right)
\end{aligned}
$$

mit den Abkürzungen $B_{1}=4 A_{1}-A_{4}, \quad B_{2}=-2 A_{2}+A_{5}, \quad B_{3}=2 A_{2}$,

$$
a_{r+r^{\prime}}^{j}=\frac{3}{64(2 \pi)^{4}} \frac{b^{2} L\left(c^{\mathrm{T}}\right)^{4}}{\left(c^{j}\right)^{7} \hbar^{4}}(K T)^{r+r^{\prime}+3}
$$

und $\quad \Delta^{j j^{\prime}}= \begin{cases}0 & \text { für } j=\mathrm{L}, j^{\prime}=\mathrm{T} \text { oder umgekehrt } \\ 1 & \text { in allen anderen Fällen. }\end{cases}$

11 H. Bross, Z. Phys., in Vorbereitung.

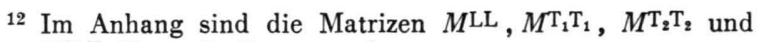
$M^{\mathrm{T}} \mathrm{T}_{2}$ für große $R_{i i^{\prime}}$ angegeben. 


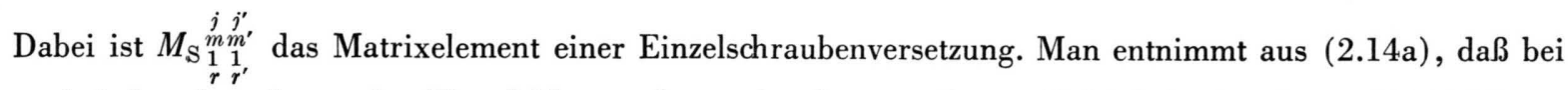
statistischer Anordnung der Gitterfehler - der zweite Summand von (2.14a) ist in diesem Fall Null die Beiträge der einzelnen Fehlstellen zur Gesamtmatrix additiv sind. $C\left(j, j^{\prime} ; m, m^{\prime} ; \alpha, \beta ; k l\right)$ sind gewöhnliche Zahlen. Durch die Größen $\mathfrak{J}\left(m+m^{\prime}, r+r^{\prime}, k, l ; b_{i i^{\prime}}\right)$ haben wir das Integral

$$
\begin{aligned}
\Im\left(m+m^{\prime}, r+r^{\prime}, k, l ; b_{i i^{\prime}}^{i}\right)= & \int_{0}^{\infty} \frac{e^{z}}{\left(e^{z}-1\right)^{2}} z^{r+r^{\prime}+3} \mathrm{~d} z \\
& \cdot \int_{0}^{\pi} \int_{0}^{2 \pi} J_{m+m^{\prime}}\left(b_{i i^{\prime}}^{j} z \sin \vartheta \sin \psi / 2\right)(\sin \vartheta)^{m+m^{\prime}+1} \cos ^{2 k} \vartheta \cos l \psi \mathrm{d} \vartheta \mathrm{d} \psi
\end{aligned}
$$

abgekürzt. Dabei sind

$$
b_{i i^{\prime}}^{j}=2\left[K T /\left(\hbar c^{j}\right)\right] R_{i i^{\prime}} \quad(2.14 \mathrm{e}) \quad \text { und } \quad z=\hbar \omega_{\mathrm{k}}^{j} /(K T) .
$$

Der Exponentialfaktor im Integranden von (2.14d) rührt von dem Produkt der mittleren Besetzungszahlen des thermischen Gleichgewichts her. Da nach (2.5a und b) und (2.6d) $\bar{W}_{\mathrm{Sf}}^{j} \mathrm{ft}^{j^{\prime}}$ proportional zu $\left(\omega_{\mathrm{f}}^{j}\right)^{0}$ ist, geht nach (2.11a) die Frequenz mit der Potenz $r+r^{\prime}+3$ in das Integral (2.14d) ein. In der Näherung $n=1$ kann $k$ die Werte $0,1,2,3$ und 4 annehmen, während $l$ gleich $0,1,2$ oder 3 ist.

$$
\text { c) Auswertung des Integrals } \mathfrak{J}\left(m+m^{\prime}, r+r^{\prime}, k, l ; b \underset{i i^{\prime}}{j}\right)
$$

$m+m^{\prime}$ ist - in der Näherung $n=1-0$ oder 2, wie oben schon erwähnt wurde. Es läßt sich zeigen, daß $\mathfrak{J}\left(2, r+r^{\prime}, k, l ; b_{i i^{\prime}}^{i}\right)$ auf Integrale mit $m+m^{\prime}=0$ zurückgeführt werden kann. Es ist hierzu die Bessec-Funktion $J_{2}(x)$ in bekannter Weise durch $J_{2}(x)=J_{0}(x)+2 \cdot \mathrm{d}^{2} J_{0}(x) / \mathrm{d} x^{2}$ darzustellen. Durch partielle Integration erhält man dann die Beziehung:

$$
\mathfrak{\Im}\left(2, r+r^{\prime}, k, l ; b_{i i^{\prime}}^{j}\right)=-\mathfrak{\Im}\left(0, r+r^{\prime}, k, l ; b_{i i^{\prime}}^{j}\right)+\frac{2 k+3}{2 k+1} \Im\left(0, r+r^{\prime}, k+1, l ; b_{i i^{\prime}}^{j}\right) .
$$

Wir können uns deshalb darauf beschränken, nur das Integral $\mathfrak{\Im}\left(0, r+r^{\prime}, k, l ; b_{i i^{\prime}}^{j}\right)$ zu berechnen.

a) Fall I: $i=i^{\prime}$, d. h. $b_{i i^{\prime}}^{j}=0$. Dieser Fall soll nur deshalb kurz untersucht werden, weil er ohne großen rechnerischen Aufwand die maximalen Werte liefert, die das Integral $\mathfrak{s}\left(m+m^{\prime}, r+r^{\prime}, k, l ; b_{i i^{\prime}}^{j}\right)$ annehmen kann (bei statistischer Verteilung der Versetzungen treten nur Integrale mit $b_{i i^{\prime}}^{j}=0$ auf). Da die BesseLFunktionen $J_{v}(x)$ bei $x=0$ nur dann von Null verschieden sind, wenn $v=0$ ist, folgt aus (2.14d)

$$
\Im\left(m+m^{\prime}, r+r^{\prime}, k, l ; 0\right)=\delta_{m+m^{\prime}, 0} \cdot \int_{0}^{\infty} \frac{e^{z}}{\left(e^{z}-1\right)^{2}} z^{r+r^{\prime}+3} \mathrm{~d} z \int_{0}^{\pi} \sin \vartheta \cos ^{2 k} \vartheta \mathrm{d} \vartheta \int_{0}^{2 \pi} \cos l \psi \mathrm{d} \psi .
$$

Die beiden Winkelintegrale lassen sich sofort ausführen:

$$
\Im\left(m+m^{\prime}, r+r^{\prime}, k, l ; 0\right)=\frac{4 \pi}{2 k+1} \delta_{l, 0} \delta_{m+m^{\prime}, 0} \int_{0}^{\infty} \frac{e^{z}}{\left(e^{z}-1\right)^{2}} z^{r+r^{\prime}+3} \mathrm{~d} z .
$$

Nach partieller Integration läßt sich (2.16b) auf ein bekanntes Exponentialintegral zurückführen. Man erhält

$$
\mathfrak{\Im}\left(m+m^{\prime}, r+r^{\prime}, k, l ; 0\right)=\frac{4 \pi}{2 k+1} \delta_{l, 0} \delta_{m+m^{\prime}, 0}\left(r+r^{\prime}+3\right) ! \zeta\left(r+r^{\prime}+3\right)
$$

Dabei ist $\zeta$ die Rremannsche Zeta-Funktion.

$\beta$ ) Fall II : $i \neq i^{\prime}$. Da der Integrand von $(2.14 \mathrm{~d})$ mindestens wie $z^{1}$ gegen Null geht $\left(r+r^{\prime} \geqq 0\right)$, können wir die Exponentialfunktion entwickeln:

$$
\int_{0}^{\infty} \frac{e^{z}}{\left(e^{z}-1\right)^{2}} f(z) \mathrm{d} z=-\int_{0}^{\infty} \frac{\mathrm{d}}{\mathrm{d} z}\left(\frac{1}{e^{z}-1}\right) f(z) \mathrm{d} z=-\int_{0}^{\infty} \frac{\mathrm{d}}{\mathrm{d} z}\left(\sum_{s=1}^{\infty} e^{-s z}\right) f(z) \mathrm{d} z .
$$

Setzen wir diese Umformung in (2.14d) ein und differenzieren entsprechend der Potenz von $z\left(r+r^{\prime}+3\right)$. mal nach $s$, so wird: 


$$
\begin{array}{r}
\Im\left(0, r+r^{\prime}, k, l ; b_{i i^{\prime}}^{j^{\prime}}\right)=-4 \sum_{s=1}^{\infty}(-1)^{r+r^{\prime}} s \frac{\mathrm{d}^{r+r^{\prime}+3}}{\mathrm{~d} s^{r+r^{\prime}+3}} \int_{0}^{\infty} e^{-s z} \mathrm{~d} z \\
\cdot \int_{0}^{\pi / 2} \int_{0}^{\pi} J_{0}\left(b_{i i^{\prime}}^{i_{1}} z \sin \vartheta \sin \psi\right) \sin \vartheta \cos ^{2 k} \vartheta \cos 2 l \psi \mathrm{d} \vartheta \mathrm{d} \psi,
\end{array}
$$

wobei die Differentiation und die Summation bezüglich $s$ nicht vertauscht werden darf. Die Integration über $\vartheta$ ergibt ${ }^{13}$

$$
\begin{aligned}
i\left(k, l ; b_{i i^{\prime}}^{j} z\right) & =\int_{0}^{\pi / 2} \int_{0}^{\pi} J_{0}\left(b_{i i^{\prime}}^{j} z \sin \vartheta \sin \psi\right) \sin \vartheta \cos ^{2 k} \vartheta \cos 2 l \psi \mathrm{d} \vartheta \mathrm{d} \psi \\
& =2^{k-1 / 2} \Gamma\left(k+{ }^{1 / 2}\right) \int_{0}^{\pi} \cos 2 l \psi \frac{J_{k+1 / 2}\left(b_{i i^{\prime}}^{j} z \sin \psi\right)}{\left(b_{i i^{\prime}}^{j} z \sin \psi\right)^{k+1 / 2}} .
\end{aligned}
$$

Mit Hilfe der Integraldarstellung der BESSEL-Funktionen

$$
J_{\nu}(y)=\frac{2(y / 2)^{\nu}}{\sqrt{\pi} \Gamma\left(\nu+{ }^{1 / 2}\right)} \int_{0}^{1} \cos (y t)\left(1-t^{2}\right)^{\nu-1 / 2} \mathrm{~d} t
$$

kann man das Integral (2.19a) über $\psi$ noch umformen:

$$
i\left(k, l ; b_{i i^{\prime}}^{j} z\right)=\frac{\Gamma(k+1 / 2)}{\sqrt{\pi} \Gamma(k+1)} \int_{0}^{1}\left(1-t^{2}\right)^{k} \mathrm{~d} t \int_{0}^{\pi} \cos \left(b_{i i^{\prime}}^{j} z \sin \psi t\right) \cos 2 l \psi \mathrm{d} \psi
$$

oder

$$
i\left(k, l ; b_{i i^{\prime}}^{j} z\right)=\frac{\sqrt{\pi} \Gamma(k+1 / 2)}{\Gamma(k+1)} \int_{0}^{1} J_{2 l}\left(b_{i i^{\prime}}^{j} z t\right)\left(1-t^{2}\right)^{k} \mathrm{~d} t .
$$

Nun können wir die Integration über die Variable $z$ ausführen ${ }^{13}$. Es ist

$$
\int_{0}^{\infty} e^{-s z} J_{2 l}\left(b_{i i^{\prime}}^{j} z t\right) \mathrm{d} z=\frac{1}{\sqrt{s^{2}+\left(b_{i i^{\prime}}^{j} t\right)^{2}}} \frac{\left(b_{i i^{\prime}}^{j} t\right)^{2 l}}{\left(s+\sqrt{s^{2}+\left(b_{i i^{\prime}}^{j} t\right)^{2}}\right)}{ }^{2 l} .
$$

Führen wir noch die Substitution

$$
t=2 \frac{s}{b_{i i^{\prime}}^{j}} \frac{a y}{1-(a y)^{2}} \quad \text { (2.21a) } \quad \text { mit } \quad a=\left\{\frac{\left[1+\left(b_{i i^{\prime}}^{j} / s\right)^{2}\right]^{1 / 2}-1}{\left[1+\left(b_{i i^{\prime}}^{j} / s\right)^{2}\right]^{1 / 2}+1}\right\}^{1 / 2}
$$

ein, so folgt aus (2.18) mit den Gln. (2.19) bis (2.21)

$$
\begin{gathered}
\Im\left(0, r+r^{\prime}, k, l ; b_{i i^{\prime}}^{j}\right)=-4(-1)^{r+r^{\prime}} \frac{\sqrt{\pi} \Gamma\left(k+{ }^{1 / 2}\right)}{\Gamma(k+1)} \\
\cdot \sum_{s=1}^{\infty} s \frac{\mathrm{d}^{r+r^{\prime}+3}}{\mathrm{~d} s^{r+r^{\prime}+3}} \frac{2 a^{2 l+1}}{b_{i i^{\prime}}^{j}} \int_{0}^{1} \frac{y^{2 l}\left[1-\left(\frac{2\left(s / b_{i i^{\prime}}^{j}\right) a y}{1-a^{2} y^{2}}\right)^{2}\right]^{k}}{1-a^{2} y^{2}} \mathrm{~d} y .
\end{gathered}
$$

Die Form von (2.22) läßt vermuten, daß die Integrale $\mathfrak{J}\left(0, r+r^{\prime}, k, l\right)$ für große $t_{i i^{\prime}}^{j}$, d. h. große Versetzungsabstände oder hohe Temperaturen wie $1 / b_{i i^{\prime}}^{i}$ gegen Null gehen, was die genauere Untersuchung im Anhang auch bestätigt. Das Integral über $y$ kann ebenfalls analytisch ausgewertet werden. Wir wollen die Integration hier jedoch nicht im einzelnen durchführen, sondern uns darauf beschränken, zwei Rekursionsformeln anzugeben, mit denen das Integral zu lösen ist. Alle Integrale (2.22) haben die Form ${ }^{14}$

$$
g(f, l)=\frac{a^{2 l} 2^{2 f+1}}{w^{2 f+1}} \int_{0}^{1} \frac{y^{2(l+f)}}{\left(1-a^{2} y^{2}\right)^{2 f+1}} \mathrm{~d} y, \quad(2.23 \mathrm{a}) \quad \text { wobei } \quad w=\left[1+\left(\frac{b_{i i^{\prime}}^{j}}{s}\right)^{2}\right]^{1 / 2}+1 \text { ist. }
$$

Mit partieller Integration bestätigt man die Rekursionsformel

$$
g(f, l+1)=\frac{1}{2 l-2 f+1}\left\{(2 l+2 f+1) g(f, l)-\frac{2}{w} a^{2 l}\right\} .
$$

13 Magnus-Oberhettinger, Formeln und Sätze für die speziellen Funktionen der mathematischen Physik, Springer-Verlag, Berlin 1948. 
Die restlichen Integrale $\quad g(f, 0)=\frac{2^{2 f+1}}{w^{2 f+1}} \int_{0}^{1} \frac{y^{2 f}}{\left(1-a^{2} y^{2}\right)^{2 f+1}} \mathrm{~d} y$

lassen sich mit der ebenfalls durch partielle Integration zu bestätigenden Rekursionsformel

$j(p, q)=\frac{1}{2(q-1)}\left\{\frac{1}{\left(1-a^{2}\right)^{q-1}}+(2 q-p-3) j(p, q-1)\right\}(2.26)$ mit $j(p, q)=\int_{0}^{1} \frac{x^{p}}{\left(1-a^{2} x^{2}\right)^{q}} \mathrm{~d} x$

auf Integrale der Form

$$
\int_{0}^{1} \frac{y^{2 f}}{1-a^{2} y^{2}} \mathrm{~d} y=-\frac{1}{a^{2 f}}\left\{\sum_{\nu=0}^{f-1} \frac{a^{2 v}}{2 v+1}-\frac{1}{2 a} \ln \left(\frac{1+a}{1-a}\right)\right\}
$$

zurückführen. Führt man schließlich noch die Differentiationen nach $s$ aus, so ergeben sich die folgenden Ausdrücke:

$\mathfrak{\Im}(0,0,0, l)=4 \pi \sum_{s=1}^{\infty} \frac{a^{2 l}}{s^{3}}\left\{\frac{2 l u\left(2 l u+2 u^{2}+3\right)+3}{u^{5}}+\frac{2}{u}+\frac{1}{u^{3}}\right\}$,

$\mathfrak{\Im}(0,0,1, l)=4 \pi \sum_{s=1}^{\infty} \frac{a^{2 l}}{s^{3}}\left\{\frac{1}{u^{3}}+\frac{2 l}{u^{2}}+\frac{1}{u}\right\}$,

$\mathfrak{\Im}(0,0,2, l)=12 \pi \sum_{s=1}^{\infty} \frac{1}{s^{3}}\left\{\frac{a^{2 l}}{u}+3 g(2, l)\right\}$,

$\mathfrak{\Im}(0,0,3, l)=30 \pi \sum_{s=1}^{\infty} \frac{1}{s^{3}}\{5 g(3, l)-3 g(2, l)\}$,

$\mathfrak{\Im}(0,0,4, l)=\quad \frac{105 \pi}{2} \sum_{s=1}^{\infty} \frac{1}{s^{3}}\{10 g(3, l)-3 g(2, l)-7 g(4, l)\} ;$

$\mathfrak{\Im}(0,1,0, l)=4 \pi \sum_{s=1}^{\infty} \frac{a^{2 l}}{s^{4}}\left\{\frac{15}{u^{7}}+\frac{30 l}{u^{6}}+\frac{24 l^{2}}{u^{5}}+\frac{8 l^{3}+10 l}{u^{4}}+\frac{12 l^{2}+3}{u^{3}}+\frac{12 l}{u^{2}}+\frac{6}{u}\right\}$,

$\Im(0,1,1, l)=4 \pi \sum_{s=1}^{\infty} \frac{a^{2 l}}{s^{4}}\left\{\frac{3}{u^{5}}+\frac{6 l}{u^{4}}+\frac{4 l^{2}+2}{u^{3}}+\frac{6 l}{u^{2}}+\frac{3}{u}\right\}$,

$\mathfrak{\Im}(0,1,2, l)=12 \pi \sum_{s=1}^{\infty} \frac{1}{s^{4}}\left\{3 g(2, l)+\frac{a^{2 l}}{u^{3}}+2 l \frac{a^{2 l}}{u^{2}}\right\}$,

$\mathfrak{\Im}(0,1,3, l)=30 \pi \sum_{s=1}^{\infty} \frac{1}{s^{4}}\left\{3 g(2, l)-15 g(3, l)+2 \frac{a^{2 l}}{u}\right\}$,

$\mathfrak{\Im}(0,1,4, l)=\quad \frac{105 \pi}{2} \sum_{s=1}^{\infty} \frac{1}{s^{4}}\{3 g(2, l)-30 g(3, l)+35 g(4, l)\} ;$

$\Im(0,2,0, l)=4 \pi \sum_{s=1}^{\infty} \frac{a^{2 l}}{s^{5}}\left\{\frac{105}{u^{9}}+\frac{210 l}{u^{8}}+\frac{180 l^{3}-30}{u^{7}}+\frac{80 l^{3}+10 l}{u^{6}}+\frac{16 l^{4}+56 l^{2}+9}{u^{5}}+\frac{32 l^{3}+40 l}{u^{4}}\right.$

$$
\left.+\frac{48 l^{2}+12}{u^{3}}+\frac{48 l}{u^{2}}+\frac{24}{u}\right\}
$$

$\mathfrak{J}(0,2,1, l)=4 \pi \sum_{s=1}^{\infty} \frac{a^{2 l}}{s^{5}}\left\{\frac{15}{u^{7}}+\frac{30 l}{u^{6}}+\frac{24 l^{2}+6}{u^{5}}+\frac{8 l^{3}+22 l}{u^{4}}+\frac{20 l^{2}+7}{u^{3}}+\frac{24 l}{u^{2}}+\frac{12}{u}\right\}$,

$\Im(0,2,2, l)=12 \pi \sum_{s=1}^{\infty} \frac{a^{2 l}}{s^{5}}\left\{\frac{3}{u^{5}}+\frac{6 l}{u^{4}}+\frac{4 l^{2}+2}{u^{3}}+\frac{6 l}{u^{2}}+\frac{3}{u}\right\}$,

$\Im(0,2,3, l)=30 \pi \sum_{s=1}^{\infty} \frac{1}{s^{5}}\left\{30 g(3, l)-\frac{4 a^{2 l}}{u}+\frac{2 a^{2 l}}{u^{3}}+4 l \frac{a^{2 l}}{u^{2}}\right\}$,

$\mathfrak{\Im}(0,2,4, l)=$

$$
\frac{105 \pi}{2} \sum_{s=1}^{\infty} \frac{1}{s^{5}}\left\{60 g(3, l)-140 g(4, l)+\frac{8 a^{2 l}}{u}\right\}
$$


mit der Abkürzung

$$
u=\left[1+\left(b_{i i^{\prime}}^{j} / s\right)^{2}\right]^{1 / 2} .
$$

Die Summen (2.29) wurden in Abhängigkeit vom Parameter $b_{i i^{\prime}}^{j}$ mit der elektronischen Rechenmaschine Z 22 mit der Genauigkeit von 1\% berechnet. In Abb. 1 sind, um von der charakteristischen Form der $\mathfrak{\Im}\left(0, r+r^{\prime}, k, l\right)$ einen Eindruck zu vermitteln, die Funktionen $\mathfrak{\Im}(0,0,3,0)$ und $\mathfrak{\Im}(0,0,3,1)$ über $b_{i i^{\prime}}^{j}$ aufgetragen. Für sehr kleine $b_{i i^{\prime}}^{j}$ sind alle $\mathfrak{\Im}\left(0, r+r^{\prime}, k, l\right) \quad(l>0)$ proportional zu $\left(b_{i i^{\prime}}^{j}\right)^{2}$, während im Unendlichen die Kurven das schon angedeutete Verhalten proportional zu $1 / b_{i i^{\prime}}^{j}$ zeigen. Mit zunehmenden $k$ und $l$ nehmen die Funktionswerte $\mathfrak{\Im}\left(0, r+r^{\prime}, k, l ; b\right)$ ab, was ja auch unmittelbar aus Gl. (2.14d) abzulesen ist.

\section{d) Die Wärmeleitfähigkeit}

Führen wir zunächst die Abkürzungen

$$
A=\frac{3}{32(2 \pi)^{4}} \frac{b^{2} L}{\left(c^{\mathrm{T}}\right)^{3} \hbar^{4}}(K T)^{r+r^{\prime}+3}
$$

und

$$
\underset{\substack{1 \\ m_{1}^{m} m^{\prime} \dot{m}^{\prime} \\ r+r^{\prime}}}{\substack{m^{\prime} \\ c^{\prime}}}\left(\frac{c^{\mathrm{T}}}{c^{j}}\right)^{7} \sum_{\alpha} \sum_{\beta} \sum_{k} \sum_{l} \frac{B_{\alpha} B_{\beta}}{B_{3}{ }^{2}} C\left(j, j^{\prime} ; m, m^{\prime} ; \alpha, \beta ; k, l\right) \Im\left(m+m^{\prime}, r+r^{\prime}, k, l ; b_{i i^{\prime}}^{j}\right)
$$

ein, so folgt aus $(2.14 \mathrm{a})$

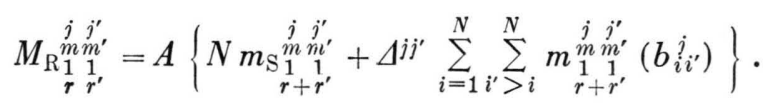

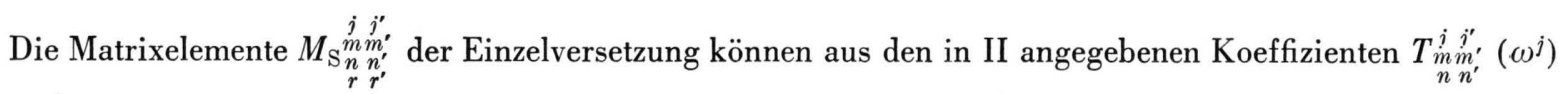
nach

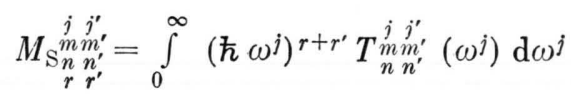

berechnet werden. Dort (II) wurde gezeigt, daß nur die Matrixelemente $T \underset{n}{m} \underset{n}{j} m_{n^{\prime}}^{j^{\prime}}$ von Null verschieden sind, was auch aus Gl. (2.11a) unmittelbar abzulesen ist. Bei der Einzelversetzung ist ja für den Anordnungsfaktor $F$ der Wert 1 zu setzen und die Integration über $\varphi$ in (2.1la) ergibt dann $2 \pi \delta_{m^{\prime},-m}$. Mit (2.34) und den Ergebnissen von II erhält $\operatorname{man}^{15}$ :

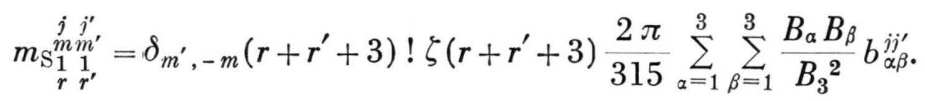

Als Beispiel für die Abhängigkeit der Matrixelemente von $b_{i i^{\prime}}^{j}$ haben wir in Abb. $2-m_{1}^{T_{1} T_{2}}{ }_{1}^{T_{2}}$ und $+m_{1}^{T_{1} T_{2}}$ für Kupfer über $b_{i i^{\prime}}^{j}$ aufgetragen ${ }^{16}$. Das Matrixelement $m_{1}^{T_{1} T_{2}}{ }_{1}^{1-1}$ ist negativ und hat bei $b_{i i^{\prime}}^{j}=0$ den Wert

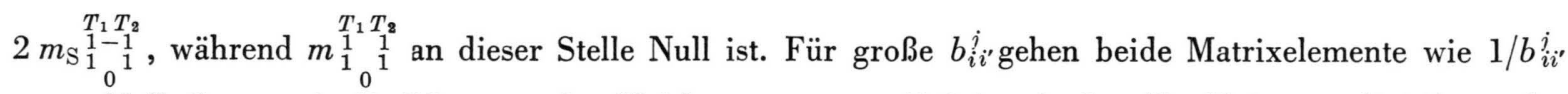
gegen Null. Setzen wir die Lösungen der Gleichungssysteme (2.9a) mit den Koeffizienten (2.33) in die Ausdrücke (2.10a) für die Leitfähigkeitskomponenten ein, so erhalten wir

$$
\varkappa_{\iota}=\frac{V_{0}}{L} \frac{K c^{\mathrm{T}}}{b^{2}} \frac{\tilde{T}^{2}}{R^{2}} \tilde{\varkappa_{\iota \iota}}(\tilde{T}) \quad(\iota=1,2) .
$$

Dabei ist die dimensionslose Größe $\tilde{\varkappa}_{u}(\tilde{T})$ - die reduzierte Wärmeleitfähigkeit - durch

$$
\tilde{\varkappa}_{u}=\frac{32}{3} \frac{1}{(2 \pi)^{2}} \sum_{\substack{j j^{\prime} \\ r r^{\prime}}} n_{r}^{j}\left(\tilde{m}_{(-1) \iota}^{-1}\right) \underset{r r^{\prime}}{j n_{r^{\prime}}} n_{r^{\prime}}^{j^{\prime}} \text { definiert, }
$$

\footnotetext{
15 Die Zahlenwerte $b_{\alpha \beta}^{i j^{\prime}}$ wurden in II für Kupfer und Germanium angegeben. 16 Die hier verwendeten Materialkonstanten für Kupfer sind
in II angegeben.
} 


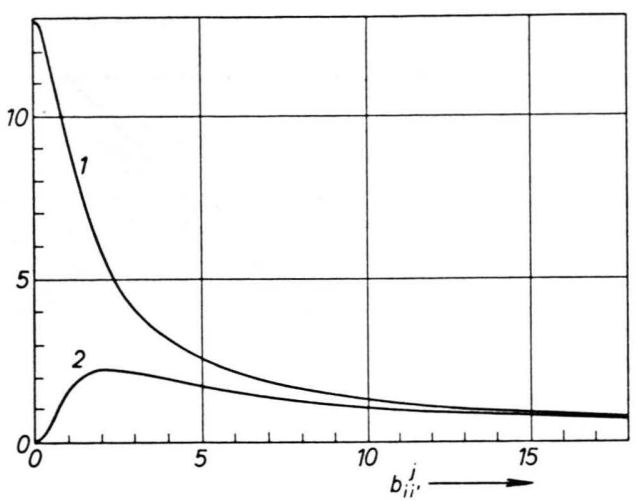

Abb. 1. Integrale $\mathfrak{I}(0,0,3,0)$ (Kurve 1$)$ und $\mathfrak{I}(0,0,3,1)$ (Kurve 2) als Funktion von $b_{i i^{\prime}}$.

Abb. 2. Matrixelemente $-m_{1}^{T_{1} T_{2}} T_{0}^{1}\left(\right.$ Kurve 1) und $m_{1}^{T_{1} T_{2}^{1}}$ (Kurve 2) für Kupfer.

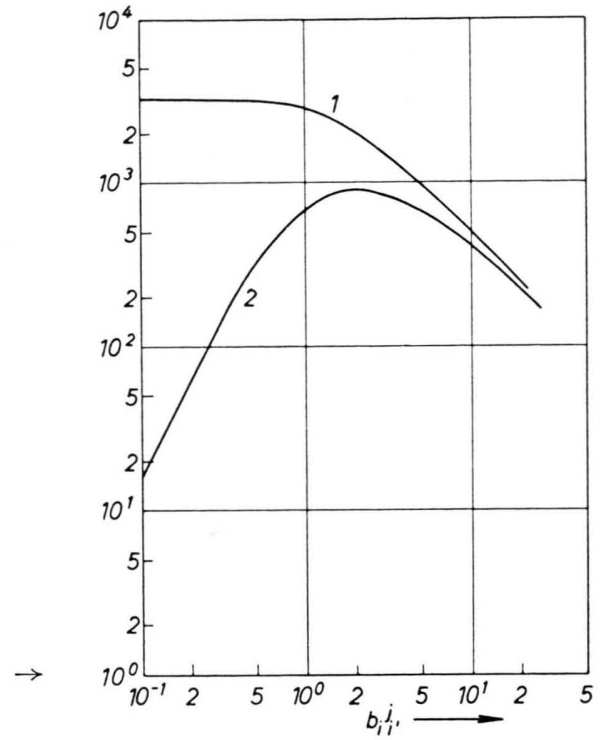

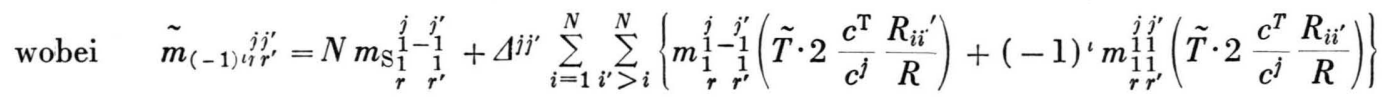

ist. $\tilde{\varkappa}_{u}$ ist außer von der reduzierten Temperatur

$$
\tilde{T}=\frac{K T}{\hbar c^{\mathrm{T}}} R
$$

wobei $\quad \frac{1}{R}=\sum_{i \neq i^{\prime}} \frac{1}{R_{i i^{\prime}}}$

ist, auch vom Verhältnis der elastischen Konstanten und von der speziellen Anordnung der Versetzungen abhängig. Ist keine Richtung im Festkörper vor der anderen ausgezeichnet, so kann die willkürlich gewählte $z$-Achse jede beliebige Orientierung haben. In diesem Fall kann ein mittlerer thermischer Widerstand

$$
\bar{\varrho}=\frac{1}{3}\left(\varrho_{11}+\varrho_{22}\right) \quad\left(\varrho_{33}=0\right)
$$

und eine mittlere Wärmeleitfähigkeit

definiert werden.

$$
\bar{\varkappa}=1 / \varrho
$$

e) Zahlenwerte der Wärmeleitfähigkeit in Kupfer für verschiedene Anordnungen von parallelen Schraubenversetzungen

Es wurden drei verschiedene Versetzungsanordnungen untersucht:
A: 6 äquidistante Versetzungen entlang der positiven $x$-Richtung;

B: 6 Versetzungen, von denen eine im Ursprung des Koordinatensystems fest verankert sein soll. Auf die Gruppe wirke entlang der negativen $x$ Achse eine homogene Spannung $\tau_{0}$;

C: 7 Versetzungen, wobei die beiden äußersten bei $x=-D / 2$ und $x=+D / 2$ fest verankert sein sollen $(D$ : Abstand der beiden festgehaltenen Versetzungen).

In allen Fällen sind die Versetzungslinien parallel zur $z$-Richtung. Die Anordnungen B und C wurden nach der von Eshelby, Frank und Nabarro angegebenen Polynommethode bestimmt ${ }^{17}$. Danach sind die Orte der "freien" Schraubenversetzungen entlang der $x$-Achse im Fall B gegeben durch die Nullstellen der ersten Ableitungen des LAguerrePolynoms $L_{6}\left(4 \pi \tau_{0} x / G b\right) ; G$ ist der Schubmodul und $b$ der Betrag des Burgers-Vektors. Im Fall $\mathrm{C}$ sind die Orte der "freien“ Versetzungen durch die Nullstellen der ersten Ableitungen des LegendrePolynoms $P_{6}(2 x / D)$ bestimmt. Die Nullstellen der

17 J. D. Eshelby, F. C. Frank u. F. R. N. Nabarro, Phil. Mag. 42, 351 [1951]. 
Laguerre- und Legendre-Polynome sind aus Tab. 1 für die Fälle $\mathrm{B}$ und $\mathrm{C}$ zu entnehmen.

\begin{tabular}{|c|r|c|}
\hline Nullstellen & $L_{6}^{\prime}(\xi)$ & $P_{6}^{\prime}(\xi)$ \\
\hline 1 & 0,621 & $-0,83$ \\
2 & 2,113 & $-0,468$ \\
3 & 4,610 & 0,000 \\
4 & 8,399 & $+0,468$ \\
5 & 14,257 & $+0,83$ \\
\hline
\end{tabular}

Tab. 1. Nullstellen der ersten Ableitungen der LaguerrePolynome $L_{6}(\xi)$ und der Legendre-Polynome $P_{6}(\xi)$.

Nach Gl. (2.36a) und (2.37a) erhält man mit den in II angegebenen Materialkonstanten für Kupfer

$$
T[\text { Grad Kelvin }]=0,18 \cdot 10^{-5} \frac{\tilde{T}}{R[\mathrm{~cm}]}
$$

und $\quad \varkappa_{\iota}\left[\frac{\mathrm{erg} / \mathrm{sec}}{\mathrm{cm} \mathrm{Grad}}\right]=\frac{V_{0}}{L} \frac{4,83 \cdot 10^{4}}{R^{2}\left[\mathrm{~cm}^{2}\right]} \tilde{T^{2}} \tilde{\varkappa_{u}}(\tilde{T)}$.

\section{f) Ergebnisse und Diskussion}

In Abb. 3, 4 und 5 ist die reduzierte thermische Leitfähigkeit in Kupfer für die Versetzungsanordnungen $\mathrm{A}, \mathrm{B}$ und $\mathrm{C}$ über der reduzierten Temperatur $\tilde{T}$ aufgetragen. Kurve 1 zeigt die mittlere reduzierte Wärmeleitfähigkeit $\tilde{T^{2}} \tilde{\varkappa}^{18}$. In Kurve 2 und 3

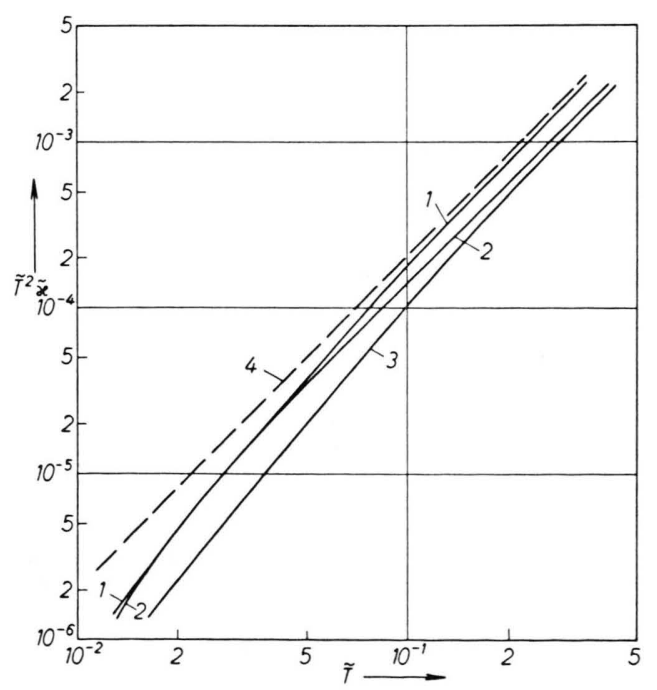

Abb. 3. Reduzierte Wärmeleitfähigkeit in Kupfer (6 Schraubenversetzungen in Anordnung A). Kurve 1: $\tilde{T}^{2} \tilde{\varkappa}, 2: \tilde{T}^{2} \tilde{\varkappa}_{11}$, 3: $\tilde{T}^{2} \tilde{\varkappa}_{22}, 4: \tilde{T}^{2} \tilde{\varkappa}_{\mathrm{S}}$.

$\left.\left.18(\tilde{T} \tilde{\tilde{\varkappa}})^{-1}=\frac{1}{3}\left\{\tilde{\left(T^{2}\right.} \varkappa_{11}\right)^{-1}+\tilde{\left(T^{2}\right.} \varkappa_{22}\right)^{-1}\right\}$.

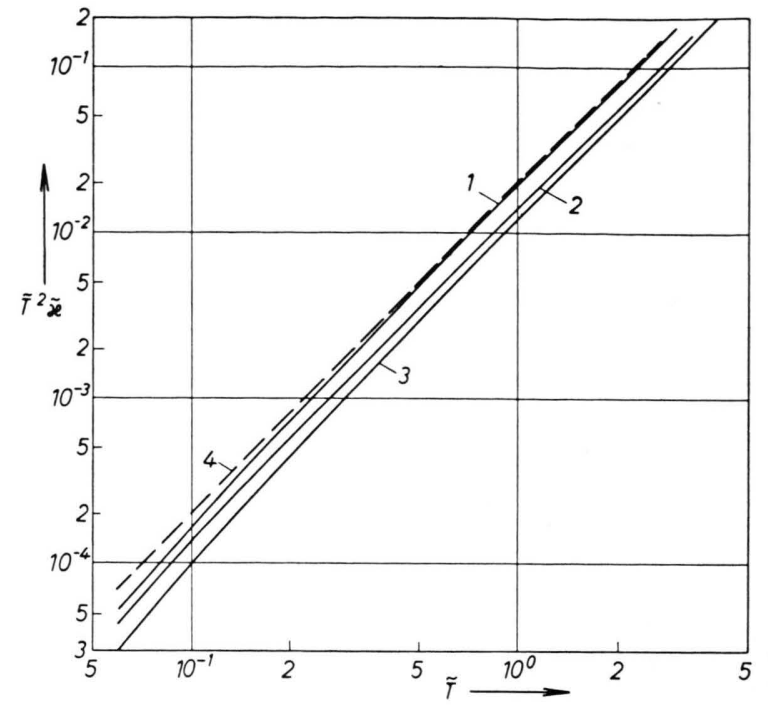

Abb. 4. Reduzierte Wärmeleitfähigkeit in Kupfer (6 Schraubenversetzungen in Anordnung B). Kurve 1: $\tilde{T}^{2} \tilde{\tilde{\varkappa}}, 2: \tilde{T}^{2} \tilde{\varkappa}_{11}$, 3: $\tilde{T}^{2} \tilde{\varkappa}_{22}, 4: \tilde{T}^{2} \tilde{\varkappa}_{\mathrm{S}}$.

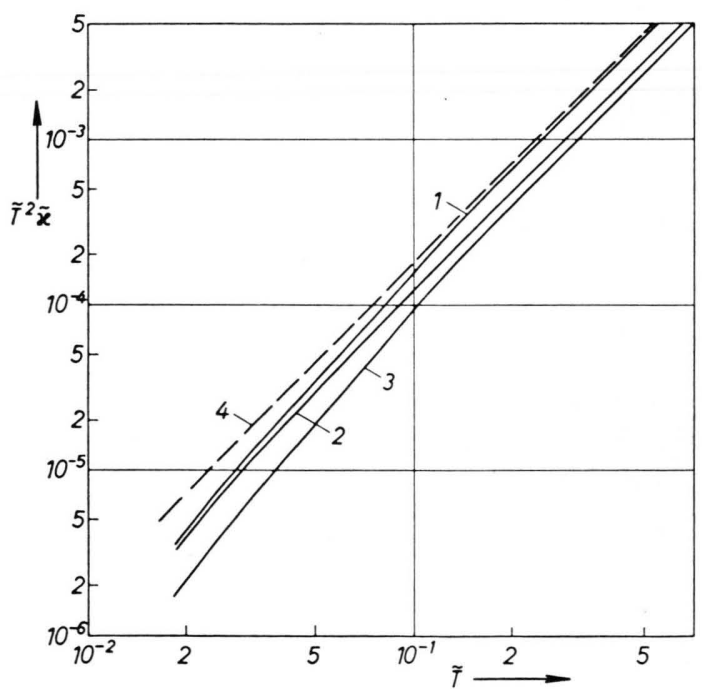

Abb. 5. Reduzierte Wärmeleitfähigkeit in Kupfer (7 Schraubenversetzungen in Anordnung C). Kurve 1: $\tilde{T^{2}} \tilde{\tilde{\varkappa}}, 2: \tilde{T}^{2} \tilde{\varkappa}_{11}$, 3: $\tilde{T}^{2} \tilde{\varkappa}_{22}, 4: \tilde{T}^{2} \tilde{\varkappa}_{\mathrm{S}}$.

ist die Komponente $\tilde{T}^{2} \tilde{\varkappa}_{11}$ bzw. $\tilde{T}^{2} \tilde{\varkappa}_{22}$ des reduzierten Leitfähigkeitstensors dargestellt. Zum Vergleich haben wir in Kurve 4 den reziproken mittleren reduzierten Wärmewiderstand $\left(\tilde{T}^{2} \bar{\varkappa}_{\mathrm{S}}\right)$ eingezeichnet, der sich bei Addition der Widerstände von Einzelversetzungen ergibt (statistische Verteilung). Am Beispiel der Anordnung B wollen wir kurz andeuten, wie man bei der Auswertung der Kurven zu ver- 
fahren hat. Es sei die homogene Spannung $\tau_{0}$ vorgegeben. Zwischen dem Abstand der $i$-ten von der $i^{\prime}$-ten Versetzung $R_{i i}$ und der Spannung besteht die Beziehung ${ }^{19}$

$$
R_{i i^{\prime}}=\frac{G b}{4 \pi \tau_{0}}\left|\xi_{i}-\xi_{i^{\prime}}\right| .
$$

Nach (2.40) und (2.37b) berechnet man mit den Werten aus Tab. 1 die zu einer bestimmten Temperatur $T$ zugeordnete reduzierte Temperatur $\tilde{T}$. Aus Abb. 4 entnimmt man dann an der Stelle $\tilde{T}$ den Wert der Funktion $\tilde{T}^{2} \tilde{\varkappa}_{u}$ und berechnet nach (2.41) die Wärmeleitfähigkeit.

Wie erwartet, ist der Wärmewiderstand einer Versetzungsgruppe bei gegebener Versetzungsdichte immer größer als derjenige bei willkürlicher Anordnung der Versetzungen. Die Differenz der Widerstände ist um so größer, je kleiner die reduzierte Temperatur, d. h. je niedriger die Temperatur $T$ und je kleiner die Versetzungsabstände sind. Während bei statistischer Verteilung der Versetzungen der Wärmewiderstand proportional zu $T^{-2}$ ist, variiert die Temperaturabhängigkeit des Gruppenwiderstands in grober Näherung zwischen $T^{-2}$ und $T^{-2,5}$. Außerdem ist im Gegensatz zur willkürlichen Anordnung bei der Gruppenanordnung entlang der $x$-Achse die Komponente des Leitfähigkeitstensors $\varkappa_{11}$ größer als $\varkappa_{22}$, weil die in Richtung $y$ laufenden Gitterwellen ein ausgedehnteres Verzerrungsfeld „sehen“ als diejenigen mit Ausbreitungsvektor in $x$-Richtung.

Durch Vergleich der asymptotischen Form der Anordnungsbeiträge (A.11) mit den Matrizen

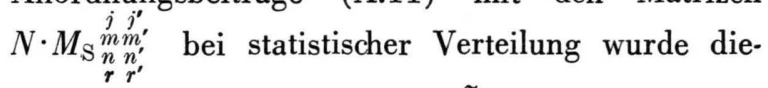
jenige reduzierte Temperatur $\tilde{T}$ bestimmt, von der ab die relative Abweichung der mittleren thermischen Leitfähigkeit $\left[\left(\bar{\varkappa}_{\mathrm{S}}-x\right) / \bar{\varkappa}_{\mathrm{S}}\right]$ kleiner als $10 \%$ ist. Es ergab sich

$$
\tilde{T} \gtrsim \tilde{T}_{\text {Grenze }}=1,19 / N,
$$

wobei $T_{\text {Grenze }}$ unabhängig von der spezifischen Anordnung ist. Aus (2.43) folgen dann die reduzierten Grenztemperaturen für die Fälle A, B und C:

$$
T_{\mathrm{A}}=T_{\mathrm{B}}=0,20, \quad T_{\mathrm{C}}=0,17 .
$$

Die absolute Grenztemperatur ist nach $(2.37 \mathrm{~b})$, (2.40) und (2.43) 20

$$
T_{\text {Grenze }}=81,49 \frac{1}{N} \cdot \sum_{i \neq i^{\prime}} \frac{1}{R_{i i^{\prime}}} .
$$

Mit (2.44) finden wir, daß oberhalb der Grenztemperaturen,

$$
T_{\mathrm{A}}=4,73{ }^{\circ} \mathrm{K}, \quad T_{\mathrm{B}}=1,62{ }^{\circ} \mathrm{K}, \quad T_{\mathrm{C}}=2,84{ }^{\circ} \mathrm{K}
$$

die relative Abweichung der mittleren Leitfähigkeit kleiner als $10 \%$ ist, wenn wir als kleinsten Versetzungsabstand $50 \mathrm{~b}$ wählen. Die Untersuchungen zeigen, daß oberhalb der reduzierten Grenztemperatur (2.43) die spezielle Art der Anordnung der Versetzungen in guter Näherung keinen Einfluß auf den Wärmewiderstand hat. Unterhalb von $T_{\text {Grenze }}$ ist bei Messungen der Wärmeleitfähigkeit auf Abweichungen vom $T^{2}$-Gesetz zu achten.

Die eingangs erwähnte Diskrepanz zwischen theoretischen und experimentellen Werten des Wärmewiderstands in Alkalihalogeniden kann durch die Berücksichtigung der Versetzungsanordnung nicht wesentlich verringert werden, auch dann nicht, wenn man annimmt, daß ein Ätzgrübchen mehrere Versetzungslinien mit Abständen von der Größenordnung $50 \mathrm{~b}$ enthält. Es muß deshalb angenommen werden, daß für den Hauptbeitrag zum Wärmewiderstand in Alkalihalogeniden ein Streumechanismus verantwortlich ist, der mit der Ätzgrübchenmethode nicht nachzuweisen ist. Dieser muß, wie die Experimente zeigen, zu einer Wärmeleitfähigkeit, die ungefähr proportional zu $T^{2}$ ist, führen. Jede Erklärung dieser Diskrepanz muß aber so geartet sein, daß sie die gute Übereinstimmung zwischen Theorie und Experiment bei Legierungen nicht verletzt.

\section{Anhang}

a) Asymptotische Form der Integrale $\Im\left(m+m^{\prime}, r+r^{\prime}, k, l ; b_{i i^{\prime}}^{j^{\prime}}\right)$

Nach $(2.14 \mathrm{~d})$ und $(2.19 \mathrm{c})$ ist $\quad \Im\left(0, r+r^{\prime}, k, l ; b_{i i^{\prime}}^{j}\right)=4 \int_{0}^{\infty} \frac{e^{z}}{\left(e^{z}-1\right)^{2}} z^{r+r^{\prime}+3} i\left(k, l ; b_{i i^{\prime}}^{j} z\right)$

19 Der Ort der $i$-ten Versetzung ist $x_{i}=\left[G \quad b /\left(4, \pi \tau_{0}\right)\right] \xi_{i}$

( $\xi_{i}=i$-te Nullstelle des Polynoms $L_{6}^{\prime}$ ).
20 Man beachte, daß $\frac{\bar{\varkappa}_{\mathrm{S}}-\bar{\varkappa}}{\overline{\chi_{\mathrm{S}}}}=\frac{\Delta \varrho_{\mathrm{I}} / \varrho \mathrm{I}}{1+\Delta \varrho_{\mathrm{I}} / \varrho \mathrm{I}}$ ist, weshalb sich der Zahlenwert von (2.44) von dem an anderer Stelle angegebenen $^{11}$ - dort wurde gefordert, da $\Delta \varrho_{\mathrm{I}} / \varrho_{\mathrm{I}} \leqq 0,1$ ist - um $10 \%$ unterscheidet. 
mit

$$
i\left(k, l ; b_{i i^{\prime}}^{j} z\right)=\frac{\sqrt{\pi} \Gamma\left(k+{ }^{1} / 2\right)}{\Gamma(k+1)} \int_{0}^{1} J_{2 l}\left(b_{i i^{\prime}}^{j} z t\right)\left(1-t^{2}\right)^{k} \mathrm{~d} t .
$$

Da der Integrand in (A.la) mindestens wie $z^{r+r^{\prime}+1}\left(r+r^{\prime} \geqq 0\right)$ gegen Null geht, verschwindet auch für sehr große $b_{i i^{\prime}}^{i}$ Werte der Beitrag zum Integral (A.la) an der unteren Grenze. Wir können deshalb in (A.1b) das Produkt

$$
B=b_{i i^{\prime}}^{j} z
$$

für $b_{i i^{\prime}}^{j} \rightarrow \infty$ als sehr groß ansehen. Führen wir in (A.lb) die Substitution

$$
x=b_{i i^{\prime}}^{j} z t=B t \quad \text { ein, }
$$

so ist $\quad i(k, l ; B)=\frac{\sqrt{\pi} \Gamma(k+1 / 2)}{\Gamma(k+1)} \frac{1}{B} \int_{0}^{B} J_{2 l}(x)\left(1-\frac{x^{2}}{B^{2}}\right)^{k} \mathrm{~d} x$

oder $\quad i(k, l ; B)=\frac{\sqrt{\pi} \Gamma\left(k+{ }^{1} / 2\right)}{\Gamma(k+1)}\left\{\frac{1}{B} \int_{0}^{B} J_{2 l}(x) \mathrm{d} x+\frac{1}{B} \sum_{\nu=1}^{k}\left(\begin{array}{l}k \\ v\end{array}\right)(-1)^{\nu} \frac{1}{B^{2 \nu}} \int_{0}^{B} J_{2 l}(x) x^{2 v} \mathrm{~d} x\right\}$.

Mit Hilfe partieller Integration kann gezeigt werden, daß die Integrale des zweiten Summanden in (A.5) mit höherer Potenz in $1 / B$ gegen Null gehen als das erste Integral. Für große $B$ ist deshalb

$$
i(k, l ; B) \sim \frac{\sqrt{\pi} \Gamma\left(k+{ }^{1} / 2\right)}{\Gamma(k+1)} \frac{1}{B} \int_{0}^{B} J_{2 l}(x) \mathrm{d} x .
$$

Die Integration in (A.6) führt man zweckmäßigerweise mit Hilfe einer Abschneidefunktion $e^{-x / B}$ aus, dann kann die obere Grenze des Integrals gleich unendlich gesetzt werden:

$i(k, l ; B) \sim \frac{\sqrt{\pi} \Gamma\left(k+{ }^{1} / 2\right)}{\Gamma(k+1)} \frac{1}{B} \int_{0}^{\infty} e^{-x / B} J_{2 l}(x) \mathrm{d} x$ (A.7a) oder $i(k, l ; B) \sim \frac{\sqrt{\pi} \Gamma(k+1 / 2)}{\Gamma(k+1)} \frac{1}{B}$.

Damit haben die Integrale $\mathfrak{\Im}\left(m+m^{\prime}, r+r^{\prime}, k, l ; b_{i i^{\prime}}^{i^{\prime}}\right)$ nach (A.1a) und (2.15) folgende asymptotische Form:

$$
\begin{aligned}
& \Im\left(0, r+r^{\prime}, k, l ; b_{i i^{\prime}}^{j^{\prime}}\right) \sim \frac{4 \sqrt{\pi} \Gamma\left(k+{ }^{1} / 2\right)}{\Gamma(k+1)}\left(r+r^{\prime}+2\right) ! \zeta\left(r+r^{\prime}+2\right) \frac{1}{b_{i i^{\prime}}^{j^{\prime}}}, \\
& \Im\left(2, r+r^{\prime}, k, l ; b_{i i^{\prime}}^{i^{\prime}}\right) \sim \frac{2 \sqrt{\pi} \Gamma\left(k+{ }^{1} / 2\right)}{\Gamma(k+2)}\left(r+r^{\prime}+2\right) ! \zeta\left(r+r^{\prime}+2\right) \frac{1}{b_{i i^{\prime}}^{j^{\prime}}} .
\end{aligned}
$$

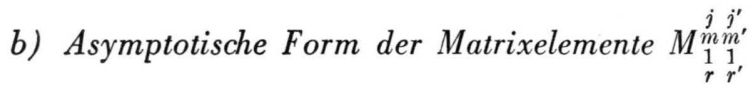

Setzt man die Ausdrücke (A.8 a und b) in (2.14a) ein, so erhält man mit

$$
D\left(r+r^{\prime}\right)=\frac{3}{32} \frac{b^{2} L}{h^{3}\left(c^{\mathrm{T}}\right)^{2}}(K T)^{r+r^{\prime}+2}\left(r+r^{\prime}+2\right) ! \zeta\left(r+r^{\prime}+2\right) \sum_{i=1}^{N} \sum_{i^{\prime}>i}^{N} \frac{1}{R_{i i^{\prime}}}
$$

für die Anordnungsbeiträge

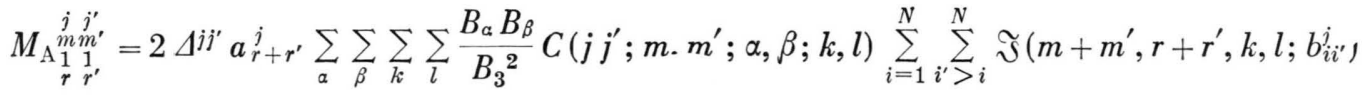

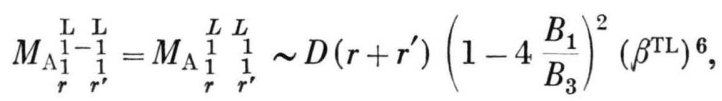

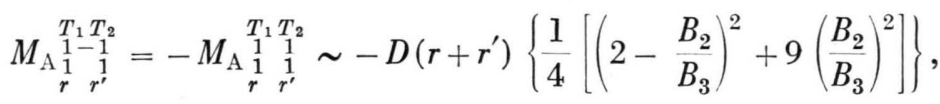

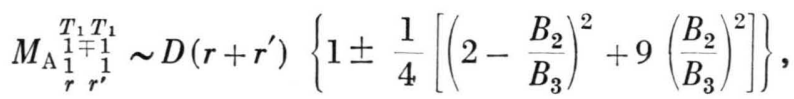

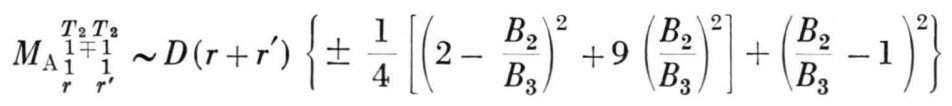




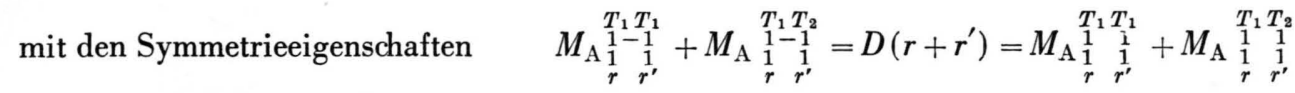

und

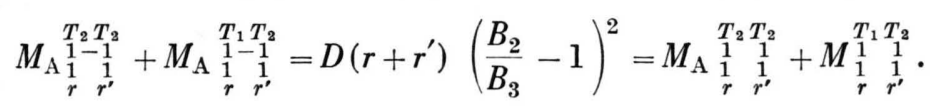

Diese asymptotischen Ausdrücke wurden auch von BRoss ${ }^{11}$ auf anderem Wege abgeleitet. Er kann mit Hilfe der Methode der stationären Phase außerdem noch die asymptotischen Werte der Matrixelemente für Übergänge zwischen longitudinalen und transversalen Wellen angeben.

Herrn Professor Dr. A. Seeger und Herrn Dozent Dr. H. Bross danke ich für die interessante Aufgabenstel. lung, für viele eingehende Diskussionen und wertvolle Ratschläge.

\title{
Der Temperaturgang der optischen und elektrischen Eigenschaften von rhomboedrischem Bor
}

\author{
Johannes Jaumann und Joachim Schnell
}

II. Physikalisches Institut der Universität Köln

(Z. Naturforschg. 20 a, 1639-1647 [1965] ; eingegangen am 5. September 1964)

\begin{abstract}
Ziel der Untersuchung war, eine Beziehung zwischen dem Absorptionsspektrum von rhomboedrischem grobkristallinen Bor und seinen elektrischen Eigenschaften im eigenleitenden Zustand $\mathrm{zu}$ suchen, wie sie vorher schon erfolgreich an Germanium ${ }^{1}$ und Silicium ${ }^{2}$ gefunden worden waren, weil dort freie Ladungsträger einen wesentlichen, mit der Wellenlänge stark wachsenden Anteil am Absorptionsspektrum haben. Bei Bor hat sich aber die Beteiligung freier Ladungsträger an der Absorption nicht überzeugend nachweisen lassen. Der langwellige Teil wird durch wenig temperaturabhängige, ziemlich scharfe Absorptionsbanden gebildet, die sicher einem Gitterschwingungsspektrum entstammen. An dem kurzwelligeren intensiven kontinuierlichen Untergrund, der mit der Temperatur stark wächst, sind sehr wahrscheinlich temperaturabhängige Kombinationsbanden der Gitterschwingungen und die Ausläufer der stark temperaturabhängigen Bandkante beteiligt, die auf eine mit wachsender Temperatur stark abnehmende Energielücke hinweist. Damit erklärt sich zwanglos die mit der Temperatur stark anwachsende und bei hohen Temperaturen, verglichen mit der Lage der Bandkante, viel zu hohe Aktivierungsenergie der Trägerzahl.
\end{abstract}

Das gesinterte, grobkristalline (Korngröße $1 / 2$ bis $1 \mathrm{~mm}$ ), porenfreie Bor der Firma $\mathrm{Starck}$, Goslar, hatte folgende spektralanalytisch festgestellten Verunreinigungen in einer Konzentration von weniger als $10^{-3} \%$ : $\mathrm{Si}, \mathrm{Mn}, \mathrm{Zn}, \mathrm{Mg}, \mathrm{Fe}, \mathrm{Cu}, \mathrm{Ca}, \mathrm{Al}$, Bi. Die C-Konzentration war sicher nicht größer als $10^{-2} \%^{3}$. Das Aussehen der Borbrocken war dunkelgrau glänzend. Drehkristallaufnahmen (wegen der groben Kristallstruktur wurde die Probe auch in der Längsachse verschoben) sind mit den von $\mathrm{HoRN}^{4}$ an rhomboedrischem Bor gemessenen Netzebenenabständen verträglich. Es handelt sich um das von SANDS und HoARD ${ }^{5}$ beschriebene „Rhombohedral Elemental Boron" mit einer Einheitszelle von 108 Atomen, $a=10,12 \AA, \alpha=65^{\circ} 28^{\prime}$. Zum Dünnschlei-

1 J. JaUmann u. R. Kessler, Z. Naturforschg. 11 a, 393 [1956].

2 R. Kessler u. J. Schnell, Z. Naturforschg. 13 a, 458 [1958].

3 Für die spektralanalytische Untersuchung danken wir Herrn Dr. K. H. SAUER, Max-Planck-Institut Düsseldorf. fen (bis $29 \mu$ mit $\pm 2 \mu$ Schwankung bei $5 \mathrm{~mm} \phi$ ) und Polieren der Proben diente Borcarbid.

\section{Absorptions- und Reflexionsspektrum}

Die Proben konnten, umgeben von einem Widerstandsöfchen, im Hochvakuum bis $700{ }^{\circ} \mathrm{C}$ erwärmt werden. Gleichzeitig konnte die elektrische Leitfähigkeit mit angedrückten Strom- und Spannungselektroden, die zugleich als Thermoelemente dienten, bestimmt werden. Die Proben waren freitragend, die dünnsten auf einem Bor-Rahmen mit Wolframfedern befestigt. Die logarithmische Auftragung der Durchlässigkeit verschiedener Proben über ihrer Dicke (Abb. 1) liefert besonders bei hohen Temperatu-

4 F. H. Honn, J. Appl. Phys. 30, 1612 [1959].

5 D. E. Sands u. J. L. Hoard, J. Amer. Chem. Soc. 79, (4), 5582 [1957]. 